\title{
Sensing and dynamics of metal ions in cell
}

\section{P251}

\section{Ratiometric imaging of cadmium ion using a novel} fluorescent sensor based on 7-amino-4-methylcoumarin Masayasu Taki ${ }^{1}$, Akio Ojida ${ }^{2}$, Itaru Hamachi ${ }^{2}$, Yukio Yamamoto ${ }^{1}$ ${ }^{1}$ Graduate School of Human and Environmental Studies,

Kyoto University, Kyoto 606-8501, Japan.

${ }^{2}$ Graduate School of Engineering, Kyoto University, Kyoto 615-8510, Japan.taki@chem.mbox.media.kyoto-u.ac.jp

Cadmium (Cd) is one of the most highly toxic heavy metal that causes a number of lesions in many organs. However, there are rare examples of fluorescent sensors which can discriminate $\mathrm{Cd}^{2+}$ from $\mathrm{Zn}^{2+}$. Herein, we describe a novel dual-excitation ratiometric probe for $\mathrm{Cd}^{2+}$, CadMQ, which employs 7-amino-4-methyl coumarin as the fluorophore.

Upon addition of $\mathrm{Cd}^{2+}$ to the HEPES-buffered solution $(50 \mathrm{mM}, \mathrm{pH}$ 7.20, 0.1 $\mathrm{M} \mathrm{KNO}_{3}$ ) of CadMQ, the excitation maximum undergoes a significant blue shift by $23 \mathrm{~nm}$ with a dissociation constant of $0.16 \mathrm{nM}$. The quantum yields of the apo and $\mathrm{Cd}^{2+}$-bound forms are determined to be 0.59 and 0.70 at neutral $\mathrm{pH}$. The fluorescence intensity of CadMQ changed with $\mathrm{pH}$ and reached a maximum at $\mathrm{pH}$ 6.3, however, the fluorescence ratios between 333 and $356 \mathrm{~nm}$ changed only slightly over the wide range of $\mathrm{pH}$. In addition, the large fluorescent shift is specific to $\mathrm{Cd}^{2+}$, and not observed in the case of $\mathrm{Zn}^{2+}$.

We have further applied CadMQ to cell imaging. CadMQ is membrane permeable and is located within the acidic compartments of the cells such as lysosome. In the case of $\mathrm{Cd}^{2+}$-exposed cells, the ratio image immediately changed (within $30 \mathrm{~s}$ ) upon addition of TPEN into the media. Decrease and increase of the fluorescence intensities at 340 and $380 \mathrm{~nm}$, respectively, induced by TPEN treatment indicate that CadMQ can probe the change of the intracellular $\mathrm{Cd}^{2+}$ levels.

\section{References}

1. Taki M, Desaki M, Ojida A, Iyoshi S, Hirayama T, Hamachi I, Yamamoto Y (2008) J Am Chem Soc 130:12564-12565

\section{P252}

Development of $\mathbf{p H}$-responsive MRI probe

Satoshi Okada, Shin Mizukami, Kazuya Kikuchi

Division of Advanced Science and Biotechnology, Graduate School of Engineering, Osaka University, Osaka 565-0871, Japan.

satoshi_okada@molpro.mls.eng.osaka-u.ac.jp

It is important to investigate in the functional roles of biomolecules not only in vitro, but also in vivo in the post-genome era. MRI is an attracting method as a molecular imaging technique, because MRI is noninvasive imaging technique that provides information in deep parts of living organism. Various MRI contrast agents have been developed for tissue $\mathrm{pH}$ imaging, because tissue $\mathrm{pH}$ values provide information about several diseases, such as kidney disorder, cancer, and so on. The switch to detect $\mathrm{pH}$ changes of relaxivity is a key factor to design MRI probes. We developed an MRI probe that detects $\mathrm{pH}$ change by conjugating a gadolinium complex with a $\mathrm{pH}-$ responsive polymer. This MRI probe changes its relaxivity by its
pH-responsive conformational changes by the intramolecular electrostatic and hydrophobic interaction (Fig. 1). Relaxivities increased as $\mathrm{pH}$ decreased, because the molecular rotation is slow at low $\mathrm{pH}$ by the intramolecular hydrophobic interaction. Steady-state fluorescence anisotropy (SSFA) measurements revealed $\mathrm{pH}$-responsive change of the molecular motion. Moreover, changes of the rotational correlation times were estimated by SSFA and fluorescence lifetime measurement.

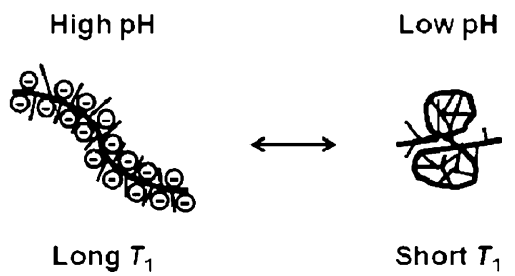

Fig. 1 pH-responsive conformational change of $\mathrm{pH}$-responsive MRI probe

\section{P253}

Synthesis and photocytotoxicity of mono-, di-, tri- and tetra-substituted glucosyl-porphyrin platinum(II) complexes for PDT drugs

Kohei Sharyo ${ }^{1}$, Shiho Hirohara', Makoto Obata ${ }^{2}$, Tsuyoshi Ando ${ }^{1}$, Masao Tanihara ${ }^{1}$

${ }^{1}$ Graduate School of Materials Science, Nara Institute of Science and Technology, Nara 630-0192, Japan.

${ }^{2}$ Graduate School of Humanities and Sciences, Nara Women's University, Nara 630-8506, Japan. s-kohei@ms.naist.jp Photodynamic therapy (PDT) is a non-invasive anticancer therapy, in which photosensitizers and light are used to generate cytotoxic reactive oxygen species in tumor cells. To clarify the effect of the

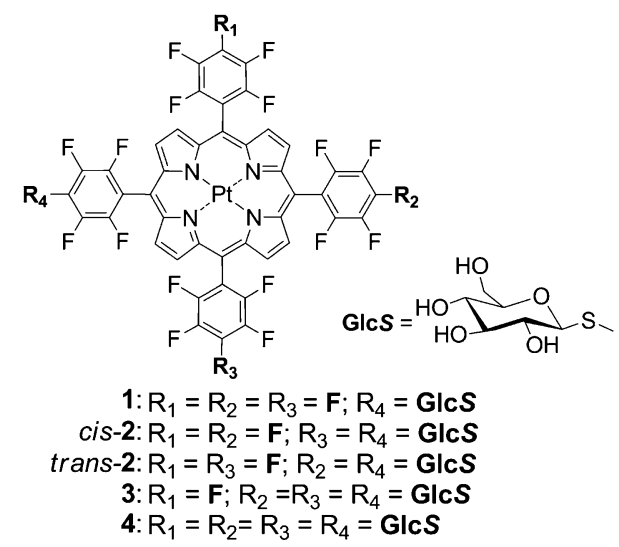

Chart 1 Glucosylated PtTFPPs 


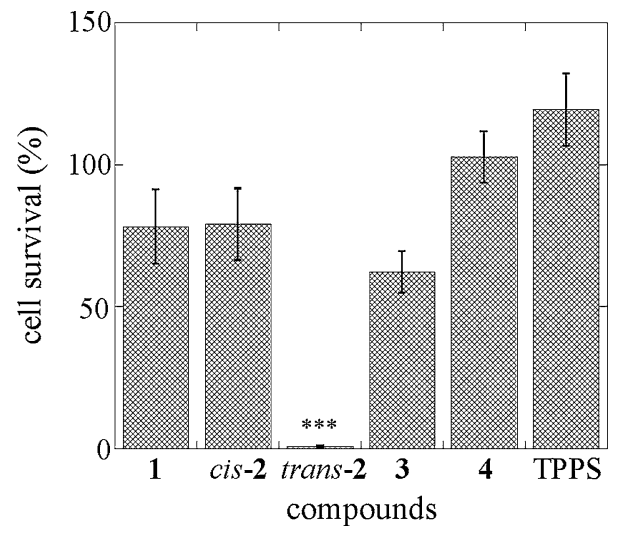

Fig. 1 Photocytotoxicity of glucosylated PtTFPPs and TPPS in HeLa cells. [Photosensitizer] $=0.5 \mu \mathrm{M} . n=6$. $* * *$ Significant difference, $p<0.001$

sugar-substitution pattern in glucosylated flurorophenylporphyrins, we synthesized flurorophenylporphyrin platinum(II) complexes (PtTFPPs) having 1-4 D-glucose molecules (1, cis-2, trans-2, 3 and 4 in Chart 1) by means of the nucleophilic substitution with thioglucoside. The photocytotoxicities of glucosylated PtTFPPs were evaluated using HeLa cells photoirradiated with a $100 \mathrm{~W}$ halogen lamp $(l>500 \mathrm{~nm})$ at a light dose of $16 \mathrm{~J} / \mathrm{cm}^{2} 24 \mathrm{~h}$ after incubation with the photosensitizers. As a result, trans-2 exerted specifically high photocytotoxicity compared with the other relating glucosylated photosensitizers (Fig. 1)

\section{P254}

\section{The diffusion of Gd-DTPA in brain ECS studied by MRI \\ Qing Xia, Hongbin Han}

State Key Laboratories of Natural and Biomimetic Drugs and Department of Chemical Biology, School of Pharmaceutical Sciences, Peking University Health Science Center, Beijing 100191, People's Republic of China. Radiology Department, Peking University the 3rd Hospital, Beijing 100191, People's Republic of China.

cjr.hanhongbin@vip.163.com

Diffusion parameters in brain which are determined by extracellular space geometry play a key role in maintaining the microenvironment of neuron. The aim of this study was to investigate the diffusion of Gd-DTPA by using MRI after the intracerebral injection of GdDTPA. Caudate nucleus was positioned by MRI scanning before the contrast injection. Two microliter of Gd-DTPA with a concentration of $5 \mu \mathrm{mol} / \mathrm{ml}$ was delivered under the guidance of stereotaxic apparatus. MRI scan were performed at 1, 3, 6, 9, 12 and $24 \mathrm{~h}$ after the injection. The MRI signal of Caudate nucleus was enhanced by the shorten relaxation effects from Gd-DTPA. The diffusion of the GdDPTA was demonstrated as the attenuation of enhanced region and the increase of the enhanced area on MRI image. The signal intensities at both the caudate nucleus and cortex regions demonstrated an exponential attenuation. The attenuation was correlated well with the increase of Gd-DTPA concentration in blood pool. The enhanced effects decreased to normal at 6-9 $\mathrm{h}$ after the injection. Transmission electron microscopy images verified that no deposition of $\mathrm{Gd}$ in the neuron. This study concluded that Gd-DTPA can diffuse in ECS, and be finally drained to blood pool. Overall, a MRI promising method using Gd-DTPA probe was developed for quantitative evaluation of materials' diffusion in ECS.

Keywords Gadolinium · ECS · Diffusion · MRI
Acknowledgments Supported by New Century Excellent Talents Program of Chinese Education Administration (NCET 06-14-01).

\section{P255}

\section{Synthesis and PDT effect of glucosylated porphyrin} metal complexes

Shiho Hirohara ${ }^{1}$, Masataka Nishida ${ }^{1}$, Kohei Sharyo ${ }^{1}$, Makoto Obata ${ }^{2}$, Tsuyoshi Ando ${ }^{1}$, Masao Tanihara ${ }^{1}$

${ }^{1}$ Graduate School of Materials Science, Nara Institute of Science and Technology, Nara 630-0192, Japan.

${ }^{2}$ Graduate School of Humanities and Sciences, Nara Women's University, Nara 630-8506, Japan. hirohara@ms.naist.jp PDT is an anti-cancer therapy, in which photosensitizers and light source are used to generate cytotoxic singlet oxygen $\left({ }^{1} \mathrm{O}_{2}\right)$ in tumor cells. In attempts to improve water-solubility and tissue selectivity, we synthesized fluorophenylporphyrin and its $\mathrm{Zn}$ (II), $\mathrm{Pd}(\mathrm{II})$ and $\mathrm{Pt}(\mathrm{II})$ complexes having D-glucose by means of nucleophilic substitution with peracetylated thioglucoside (Chart 1). Deprotection of glucopyranosyl groups was carried out by using sodium methoxide. Independent of the protection of glucopyranosyl groups, the relative quantum yield of singlet oxygen increased in the order of free-base porphyrins $<\mathrm{Zn}$ (II) complexes $<\mathrm{Pd}$ (II) complexes, which can be explained in terms of the heavy-atom effect, while the values for $\mathrm{Pt}(\mathrm{II})$ complexes were exceptionally low. In vitro photocytotoxicity of these photosensitizers were evaluated using HeLa cells photoirradiated with a $100 \mathrm{~W}$ halogen lamp $(l>500 \mathrm{~nm})$ at a light dose of $16 \mathrm{~J} /$ $\mathrm{cm}^{2} 24 \mathrm{~h}$ after incubation with the photosensitizers. Although all protected photosensitizers showed no photocytotoxicity, the deprotected free-base porphyrin and its $\mathrm{Zn}(\mathrm{II})$ and $\mathrm{Pd}(\mathrm{II})$ complexes exerted potent photocytotoxicity.

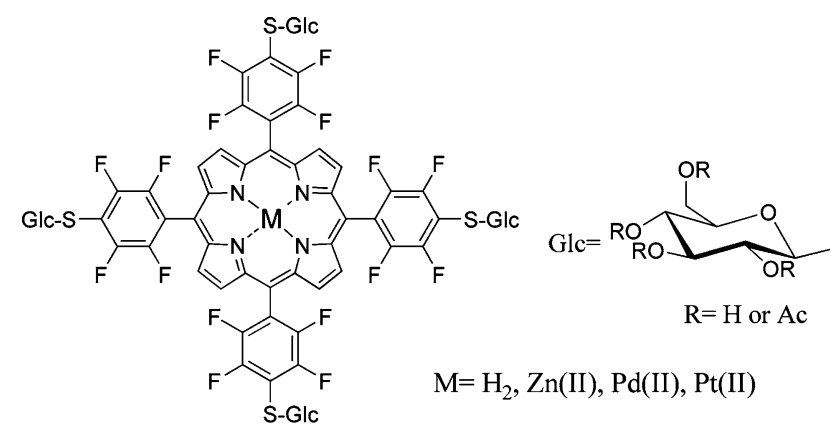

Chart 1 Fluorophenylporphyrin metal complexes

\section{P256}

Luminescent lanthanide probe for protease activities Miho Tanaka, Kazuhiro Tonai, Shin Mizukami, Kazuya Kikuchi Department of Engineering, Graduate School of Engineering, Osaka University, Osaka 565-0871, Japan.

m.tanaka@molpro.mls.eng.osaka-u.ac.jp

Lanthanide complexes show sharp and long-lifetime luminescence spectra, since the luminescence is due to the forbidden $\mathrm{f}-\mathrm{f}$ transition of the metal electron. Time-resolved fluorescence measurement enables to measure only lanthanide luminescence without short-lifetime background fluorescence such as auto-fluorescence. However, the luminescence intensity is very weak because of their small 
extinction coefficients. Therefore, "antenna" groups are used for the efficient excitation. When lanthanide ions are excited indirectly by the antenna groups, they can emit strong long-lifetime luminescence.

It has been reported that there are more than 500 kinds of proteases in human bodies, and many of them are involved in diseases. Thus, it is very important to develop fluorescent probes for detecting such protease activities. We focused on the characteristics of lanthanide luminescence and tried to detect protease activities. Some lanthanide probes are reported. However, some of them are based on photoinduced electron transfer system, which decreases the luminescence intensity after the protease reaction. Others are based on timeresolved fluorescence energy transfer (TR-FRET) system, which are laborious to be synthesized and have risk of intermolecular FRET.

Here, a fluorogenic luminescent lanthanide probe for detecting protease activities was developed. First, the suitable antenna groups were selected and one probe that can increase $\mathrm{Tb}^{3+}$ luminescence intensity by deacylation of the aminoacyl group was found. Then we designed and synthesized the antenna- $\mathrm{Tb}^{3+}$ complex with a peptide sequence that can be cleaved by a protease. When the probe was cleaved with the target protease, the time-resolved fluorescence intensity was largely increased.

\section{P257}

\section{Ratiometric $\mathrm{Zn}^{2+}$ fluorescent sensor based} on 4-amino-7-substituted-2, 1, 3-benzoxadiazole (ASBD)

\section{Weijiang He, Zhipeng Liu, Changli Zhang, Zijian Guo}

State Key Laboratory of Coordination Chemistry, Nanjing University, Nanjing 210093, People's Republic of China. heweij69@nju.edu.cn Developing $\mathrm{Zn}^{2+}$ fluorescent sensors, especially the ratiometric sensors, for clarifying the essential roles of $\mathrm{Zn}^{2+}$ in living systems, are now attract much more attention. Since $\mathrm{Zn}^{2+}$ coordination to the conjugated electron donating/withdrawing group of intramolecular charge transfer (ICT) fluorophore may lead to the emission shift demanded by ratiometric sensor. Our trial by modifying the 4-amine of ICT fluorophore 4-amino-7-nitro-2,1,3-benzoxadiazole (ANBD) as $\mathrm{Zn}^{2+}$ ionophore, however, exhibited very limited $\mathrm{Zn}^{2+}$-triggered blue emission shift (16 $\mathrm{nm}$ or even lower) of the sensor. When the 7-nitro group was replaced by 7 -sulfonamido group to form sensor SBD-TPEA, the $\mathrm{Zn}^{2+}$-triggered emission shift attained to $37 \mathrm{~nm}$, yet the free sensor and its zinc complex displayed the comparable emission intensity. The current results indicate that the fine modulation of 4,7-electron donating/withdrawing group of 2,1,3-benzoxadiazole should be an effective method to construct the novel fluorescent ratiometric $\mathrm{Zn}^{2+}$ senor.

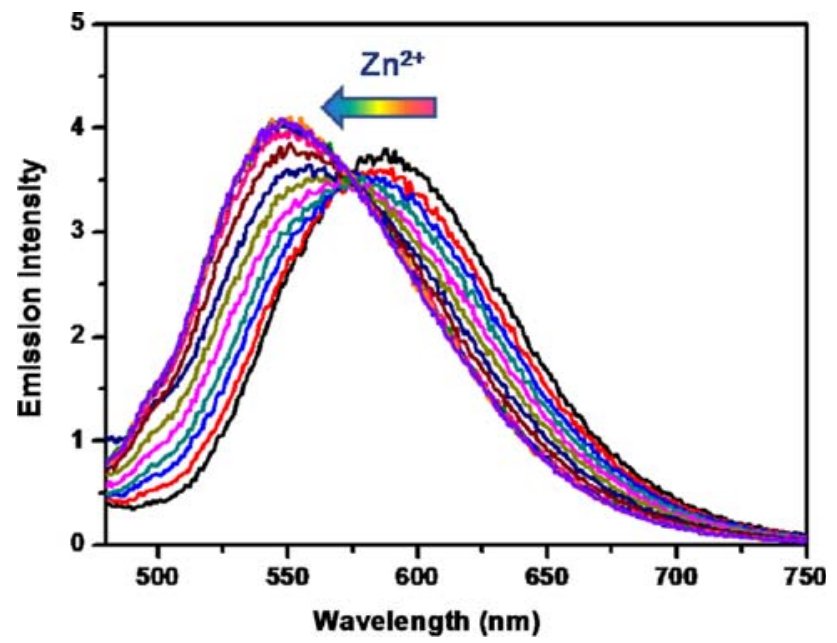

References

1. Jiang P, Guo Z (2004) Coord Chem Rev 248:205-229

2. Que EL, Domaille DW, Chang CJ (2008) Chem Rev 108:1517-1549

\section{P259}

Structure and photophysical properties of fluorescent metal sensors containing thioether binding site Shohei Iyoshi, Masayasu Taki, Yukio Yamamoto

Graduate School of Human and Environmental Studies, Kyoto University, Yoshida, Sakyo-ku, Kyoto 606-8501, Japan. shohei.iyoshi@h03.mbox.media.kyoto-u.ac.jp

Selective and sensitive fluorescent probes for metal ions have been essential tools in the field of bioinorganic chemistry. Especially, probes for soft metal ion such as $\mathrm{Ag}^{+}, \mathrm{Cu}^{+}$, and $\mathrm{Hg}^{2+}$ receive attention since these metals are known to affect human and other organisms. However, developments of fluorescent probes for these metals have been less exploited because of their fluorescence quenching abilities. Herein, we describe novel fluorescent probes containing soft thioether binding sites and report their fluorescent response properties toward these metal ions.

A rosamine-based probe containing S3O1 ligand, S3O1-TMR, has been prepared and characterized [1]. This fluorescent sensor has a negligible quantum yield $\left(4.8 \times 10^{-3}\right)$ in the absence of $\mathrm{Ag}^{+}$, whereas a significant fluorescence enhancement is observed upon complexation with $\mathrm{Ag}^{+}$under physiological conditions. The crystal structure of the silver complex with the chelator moiety of S3O1TMR reveals its trigonal planar coordination geometry in which three sulfur atoms coordinate the metal center. Although strong coordinative interaction of $\mathrm{Ag}-\mathrm{N}$ is not observed in the crystal structure, the ${ }^{1} \mathrm{H}-\mathrm{NMR}$ experiments suggest that aniline-nitrogen is likely to be associated with the $\mathrm{Ag}^{+}$center in the solution state. This may inhibit the PET process and cause the enhancement of fluorescence. Results obtained with other probes loading different types of thioether-containing binding sites will be also described.

\section{References}

1. Iyoshi S, Taki M, Yamamoto Y (2008) Inorg Chem 47:3946-3948

\section{P260}

Glyco-conjugates: ion and molecular recognition and their binding with lectins and glycosidases Atanu Mitra, Amit Kumar, Chebrolu P. Rao

Bioinorganic Laboratory, Department of Chemistry, Indian Institute of Technology Bombay Powai, Mumbai 400076, India.

atanu_mitra@iitb.ac.in

Glyco-conjugates exhibit wide range of applications in pharmaceutical industry and medicine as oral antidiabetic and inhibitors. Owing to the presence of glyco-moiety, the glyco-conjugates are expected to bind and modify the properties of lectins and glycosidases. Further, the conjugates synthesized using binding cores are suitable for ion and molecular recognition properties. Therefore, the main focus of this presentation is to show the ability of glycoconjugates in ion/molecular recognition as well as in binding and modifying the properties of lectins and glycosidases. The C2-based imino-glyco-conjugates have been thoroughly studied for their metal ion recognition property. The glycol-conjugate 1a shows selectivity towards $\mathrm{Zn}$ (II) in water while $\mathbf{1 b}$ shows response towards $\mathrm{Zn}$ (II) in methanol and $\mathrm{Cu}(\mathrm{II})$ in HEPES buffer. 1b can 


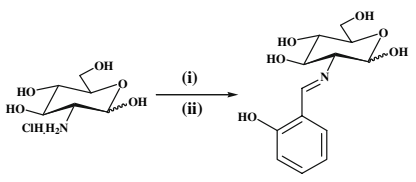

$\mathbf{a}$

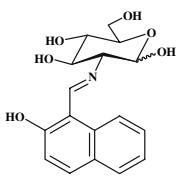

b

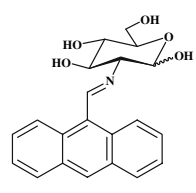

c
Fig. 1 Synthesis of $C_{2}$ based glyco-derivatives $\mathbf{1 a}, \mathbf{1 b}$ and $\mathbf{1 c}$ from glucose

also act as a naked eye sensor for Fe(III) in methanol as well as in HEPES buffer. On the other hand $\mathbf{1 c}$ is selective towards $\mathrm{Hg}(\mathrm{II})$ in methanol. The binding property of these glyco-conjugates with lectins viz. jacalin, peanut agglutinin, soybean agglutinin and pea lectin and glycosidases isolated from legume seeds were also studied. Agglutination studies were also carried out using blood cells. The details of these results will be discussed in the poster (Figs. 1, 2)

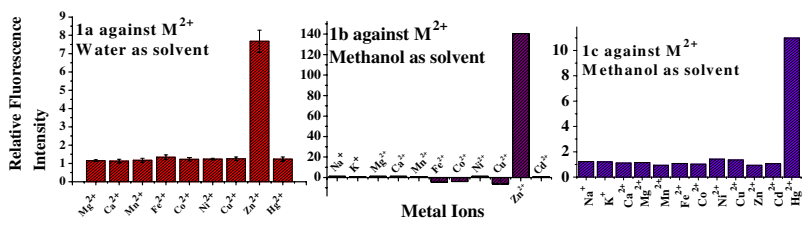

Fig. 2 Bar diagram showing selective response of $\mathbf{1 a}, \mathbf{1 b}$ and $\mathbf{1 c}$ towards different $\mathrm{M}^{2+}$ in different solvent system

\section{References}

1. Mitra A et al (2009) Tetrahedron Lett 50:776-780

2. Kumar A et al (2009) Glycoconj J. doi:10.1007/s10719-0089199-4
P262

Rhenium and 99m-technetium complexes of monosaccharide based tripodal triamines as potential radio imaging agents

${ }^{2,3}$ Michael Gottschaldt, ${ }^{2}$ Carmen Bohlender, ${ }^{4}$ Dirk Müller, ${ }^{4}$ Ingo Klette, ${ }^{4}$ Richard P. Baum, ${ }^{1}$ Shigenobu Yano, ${ }^{2,3,5}$ Ulrich S. Schubert

${ }^{1}$ Endowed Research Section, Photomedical Science, Innovative Collaboration Center, Kyoto University, Kyoto 615-8520, Japan. ${ }^{2}$ Laboratory of Organic and Macromolecular Chemistry, FriedrichSchiller- University Jena, 07743 Jena, Germany.

${ }^{3}$ Dutch Polymer Institute, John F. Kennedylaan 2, The Netherlands. ${ }^{4}$ Department of Nuclear Medicine, Zentralklinik Bad Berka GmbH, Germany.

${ }^{5}$ Laboratory of Macromolecular Chemistry and Nanoscience, Eindhoven University of Technology, 5600 MB Eindhoven, The Netherlands.

yano@icc.kyoto-u.ac.jp

Radiometal-labeled compounds are important substances for diagnosis and therapy in nuclear medicine. Because of their closely related chemical properties, but their different kind of radiation, the nuclides ${ }^{99 \mathrm{~m}} \mathrm{Tc}$ and ${ }^{186 / 188} \mathrm{Re}$ are called diagnostic and therapeutic pair. Bound to a metal centre carbohydrates are able to improve solubility, decrease toxicity and open the possibility to target saccharide-specific binding domains or metabolic pathways. In order to use these advantages, a number of radioactive metallo-labelled saccharides have recently been synthesized comprising bi- and tridentate ligands for $\operatorname{Tc}(\mathrm{I})$ and $\operatorname{Re}(\mathrm{I})$ carbonyl cores [1]. A synthetic pathway to new sugar containing tripodal triamines of the TAME type [1,1,1-tris(aminomethyl)ethane] such as Xyl-O- $\mathrm{CH}_{2} \mathrm{C}\left(\mathrm{CH}_{2} \mathrm{NH}_{2}\right)_{3}$, Glc-O- $\mathrm{CH}_{2} \mathrm{C}\left(\mathrm{CH}_{2} \mathrm{NH}_{2}\right)_{3}$ and Gal-O$\mathrm{CH}_{2} \mathrm{C}\left(\mathrm{CH}_{2} \mathrm{NH}_{2}\right)_{3}(\mathbf{5 a}-\mathbf{c})$ is presented. The triamines form complexes of the type $\left[\operatorname{Re}(\mathrm{CO})_{3} \mathrm{~L}\right] \mathrm{Cl}(\mathbf{6 a - c})$. The synthesis of the analogous radiolabeled ${ }^{99 \mathrm{~m}} \mathrm{Tc}$ complex with galactosyl appendage $7 \mathrm{c}(\mathrm{L}=\mathrm{Gal}-\mathrm{O}-$ $\left.\mathrm{CH}_{2} \mathrm{C}\left(\mathrm{CH}_{2} \mathrm{NH}_{2}\right)_{3}\right)$ was achieved for $\mathbf{5 c}$ and its stability over a period of $24 \mathrm{~h}$ could be verified by HPLC analysis, confirming the significant stability of $\mathbf{7 c}$ against histidine exchange.

Reference

1. Gottschaldt M, Koth D, Muller D, Klette I, Rau S, Gorls H, Schäfer B, Baum RP, Yano S (2007) Chem Eur J 13:10273-10280 